\title{
2011 Episodes Best Paper Award
}

IUGS Episodes Best Paper Award was established in 2010 to recognize the authors of the best original article published in any of the four annual issues of Episodes during an "annual" year.

This Award was awarded in 2011 to: Wojciech Chelmicki (Jagiellonian University, Poland), Pawel Jokiel and Piotr Moniewski (the University of Lodz, Poland), and Zdzislaw Michalczyk (Maria Curie-Sklodowska University, Poland) for their paper "Distribution, discharge and regional characteristics of springs in Poland" (Episodes, vol. 34 No. 4: December 2011, pages 244-256). 